\title{
Reward Enhances Connectivity between the Ventral Striatum and the Default Mode Network
}

Ekaterina Dobryakova ${ }^{1}$ and David V. Smith ${ }^{2}$

${ }^{1}$ Kessler Foundation, 120 Eagle Rock Ave, East Hanover, NJ, USA

${ }^{2}$ Department of Psychology, Temple University, Philadelphia, PA, USA

\section{Acknowledgments}

This work was supported, in part, by National Institutes of Health grants R21-MH113917 (DVS), R03-DA046733 (DVS), RF1-AG067011 (DVS). We note that DVS was a Research Fellow of the Public Policy Lab at Temple University during the preparation of this manuscript (2019-2020 academic year).

\section{Conflict of interest statement}

The authors have no conflicts to disclose.

\section{Data and code availability}

Statistical maps can be found on NeuroVault. Analysis code can be found on https://github.com/DVS-Lab/dmn-reward.

\section{Correspondence}

Ekaterina Dobryakova (edobryakova@kesslerfoundation.org)

David V. Smith (david.v.smith@temple.edu) 


\begin{abstract}
One of the central topics in cognitive neuroscience revolves around understanding how responses in the default mode network (DMN) relate to cognitive process and disease states. While there has been many investigations of the intrinsic patterns of activation and connectivity of the DMN with other networks at rest, i.e. when an individual is not engaged in any particular behavior, to truly understand the influence and significance of the DMN activation and connectivity, we must study it in association with a particular process. Reward processing is an integral part of goal-directed behavior that has been shown to rely on the striatum, a subcortical brain region that is connected to multiple regions of the prefrontal cortex (PFC) that belong to the DMN. Yet, it remains unclear how the DMN interacts with the striatum during reward processing. To investigate this issue, we analyzed card-guessing task data of 453 subjects from the Human Connectome Project and applied a novel network-based psychophysiological interaction analysis (nPPI) that quantified reward-dependent connectivity of the DMN. We show that only the DMN exhibits increased connectivity with the ventral striatum (VS) during the receipt of reward. This result was specific to the DMN and the strength of connectivity was associated with the personality characteristic of openness. These findings point to a novel role of the DMN during reward processing, and to the nPPI approach being able to capture a unique contribution of a collection of regions to task performance.
\end{abstract}




\section{Significance}

The default mode network (DMN) has been theorized to participate in a range of social, cognitive, and affective functions. Yet, previous accounts do not consider how the DMN contributes to other brain regions depending on psychological context, thus rendering our understanding of DMN function incomplete. We addressed this gap by applying a novel network-based psychophysiological interaction analysis to the reward task within the Human Connectome Project. We found that the experience of reward enhances task-dependent connectivity between the $\mathrm{DMN}$ and the ventral striatum. Surprisingly, the strength of connectivity was correlated with personality characteristics relating to openness. These results advance models of DMN by demonstrating how it contributes to other brain systems and how those contributions relate to individual differences. 


\section{Introduction}

A cardinal goal of neuroscience relates to characterizing how functional integration organizes discrete brain regions into cohesive networks that shape behavior (Park \& Friston, 2013). Several distinct networks have been identified in both resting and task states (Smith et al., 2009). Of these networks, the default mode network (DMN)-including the posterior medial cortex, medial prefrontal cortex, and lateral temporal-parietal regions-may be unique in its elevated baseline energy consumption (Buckner et al., 2008; Buckner \& DiNicola, 2019; Raichle et al., 2001). Activation of the DMN appears to be highest when participants are engaged in inward-directed thought and lowest when participants are engaged in externally-directed tasks requiring focused attention (Hasenkamp et al., 2012; Hayden et al., 2009; Scheibner et al., 2017). In addition, the degree of task-related deactivation has been linked to performance (Anticevic et al., 2010; Vatansever et al., 2015), suggesting a key role for the DMN in shaping behavior. Despite these advances, we still know very little about how task-related changes in DMN are linked to changes in effective connectivity with other brain regions.

Many studies have investigated the function of DMN through the lens of individual differences. These studies have helped establish that the DMN is linked to disease and psychopathology (Anticevic et al., 2012; Dobryakova et al., 2018). For instance, individuals at risk for developing Alzheimer's Disease exhibit elevated intrinsic correlations within the DMN (Filippini et al., 2009). Elevated intrinsic activity within the DMN has also been shown in schizophrenia and depression (Whitfield-Gabrieli \& Ford, 2012). These psychopathologies have also been linked to aberrant patterns of resting-state functional connectivity within the DMN. For example, individuals with schizophrenia show reduced resting-state functional connectivity between the DMN and the striatum (Wang et al., 2015). Similar effects have also been observed in individuals at risk for psychosis (Hua et al., 2019). Although altered DMN-striatal connectivity has also been observed in depression, the results are more mixed, with some studies suggesting hypoconnectivity (Bluhm et al., 2009), while other studies suggesting hyperconnectivity during resting state (Hwang et al., 2016).

Building on these findings, other studies have begun to point to a key link between the DMN and the striatum. For example, one study using physio-physiological interaction analyses 
demonstrated that the striatum interacts with the salience network and DMN (Di \& Biswal, 2014). Other studies have shown that striatal dopamine levels are linked to the magnitude of DMN deactivation during visuospatial attention (Tomasi et al., 2009) and tasks requiring cognitive flexibility (Dang et al., 2012). In addition, DMN responses have also been associated with gamma oscillations in the striatum, which may serve to facilitate switching between externally-driven and internally-driven brain states (Nair et al., 2018). While these observations illustrate some indirect relationships between the DMN and the striatum, it remains unclear whether there are direct contributions from the DMN to the striatum during a task. Observing this type of functional coupling would lend support to the emerging idea that a key function of the DMN resides with guiding goal-directed behavior and value-based decision making (Dohmatob et al., 2020).

To further investigate this possibility, we examined connectivity with the DMN during a reward processing task. We hypothesized that the psychological context of reward consumption would alter effective connectivity between the DMN and the striatum. We used data from the Human Connectome Project and applied a network-based psychophysiological interaction (nPPI) analysis (Fareri et al., 2020; Utevsky et al., 2017). This analytical approach merges canonical PPI analyses (Friston et al., 1997; McLaren et al., 2012; Smith et al., 2016) with dual-regression analysis (Filippini et al., 2009; Nickerson et al., 2017; Smith et al., 2014) to assess how networks interact with other regions as a function of task context. Our analyses focused on two key questions. First, does the receipt of reward drive connectivity between the DMN and striatum? Second, if there is reward-dependent connectivity between the DMN and striatum, is it associated with behavior and personality characteristics that are functionally relevant to reward processing?

\section{Methods}

\section{Participants}

We obtained behavioral and neuroimaging data of 495 subjects from the Human Connectome Project (HCP; www.humanconnectome.org), an open-access database aimed at collecting 
healthy participant data from over 1,200 people. Although recent work has shown that effect sizes for reward-related striatal activation in the HCP is modest with a median $d=0.40$ (Poldrack et al., 2017), we anticipated effect sizes associated with connectivity would be much smaller. Yet, even with a smaller expected effect size, we reasoned that a subsample of at least 376 participants would be sufficient for detecting small effects $(d=0.20)$ with $90 \%$ power and a two-sided paired t-test with alpha $=0.01$. While less is known about the effect sizes for individual differences in brain-behavior relationships, there is good reason to believe such effects are generally small. For example, a recent examination of 87 meta-analyses on individual differences revealed a median $r=0.19$ (Gignac \& Szodorai, 2016). Detecting this effect with $90 \%$ power and alpha $=0.01$ would require at least 405 participants. Within our subsample of 495 participants, ages ranged from 22 to 35 years old (Barch et al., 2013).

Because of technical difficulties and excessive head motion, 457 out of the 495 downloaded subjects were used in the final analysis (see below for details). Within this sample, familial relationships were unknown for 4 individuals (including on participant without NEO scores); these participants were excluded from our analysis since it was impossible to account for family structure (Winkler et al., 2014). After all exclusions, our final sample consisted of 453 participants (females $=270$ ).

\section{Behavioral Paradigm}

Participants completed a card-guessing task adapted from previous work (Delgado et al., 2000) where they had to guess whether the number on a facedown card was higher or lower than 5. Following their response, participants were presented with one of three feedback options: 1) a green up arrow indicating a correct guess resulting in a gain of \$1.00; 2) a red down arrow indicating an incorrect guess resulting in a loss of \$0.50; or 3) a gray double-headed arrow for neutral trials. Stimuli were presented in blocks of eight trials. Trial blocks were either mostly reward or mostly loss, including six of the main conditions and two randomized trials from the remaining conditions. Each condition lasted $28 \mathrm{sec}$. Two runs were completed, composed of 2 mostly reward, 2 mostly loss, and 4 randomly placed fixation blocks. The facedown card with a 
"?" was presented for up to $1.5 \mathrm{sec}$, with $1 \mathrm{sec}$ allotted for feedback and a $1 \mathrm{sec}$ ITI for the presentation of a fixation cross. All participants received standardized monetary compensations after completing the task. See Barch et al., (2013) for more details.

\section{fMRI preprocessing}

Our data preprocessing and analysis was carried out using FMRIB Software Library tools (v6.00; www.fmrib.ox.ac.uk/fsl). For the analyses in the current paper, we used the minimally preprocessed data, the end product of the volume-based fMRI pipeline, which were motion corrected and normalized to the MNI template (Glasser et al., 2013). We excluded 31 subjects whose average relative volume-to-volume motion qualified as an outlier relative to other subject; we identified outlier subject using a standard boxplot threshold (i.e., the $75^{\text {th }}$ percentile plus $1.5^{*}$ Interquartile Range (IQR); IQR for right-to-left phase encoding scan: 0.154; IQR for leftto-right phase encoding scan: 0.174$)$. We excluded an additional 7 subjects due to technical difficulties (e.g., corrupt downloads).

Further we removed the first 15 volumes to achieve steady state magnetization; and applied spatial smoothing with a $4 \mathrm{~mm}$ full-width at half-maximum (FWHM) Gaussian kernel. The output of the preprocessing was further processed with ICA-AROMA (Pruim et al., 2015), which removed motion components from the dataset and output a de-noised dataset that was further temporally filtered (high-pass temporal filtering $90 \mathrm{sec}$ ).

\section{fMRI analysis}

Statistical analysis was carried out using FILM pre-whitening with local autocorrelation correction (Woolrich et al., 2001). We constructed five models to evaluate reward processing and to evaluate reward-dependent connectivity of the 4 RSNs (corresponding to the DMN and 3 other RSNs whose regions are structurally connected to the striatum: ECN, IFPN, rFPN). All networks were taken from prior work (Smith et al., 2009). The reward and punishment blocks that lasted for $28 \mathrm{sec}$ were used as regressors and were convolved with the double-gamma hemodynamic response function (HRF). We constructed four generalized network PPI (nPPI) models (McLaren et al., 2012) with 14 regressors in each model. In addition to the reward and 
punishment regressors, following regressors of interest were included in nPPI model: RSN time course, reward x RSN time course interaction, and punishment x RSN time course interaction. For the PPI regressors, we set the minimum value of the psychological regressor to zero, and de-meaned the physiological regressor (RSN time course). To control for the influence of other networks, we included the time courses of all the other 9 RSNs as regressors of no interest. To average contrast estimates over runs within each subject, we carried out a fixed effects analysis by forcing the random effects variance to zero in FLAME (FMRIB's Local Analysis of Mixed Effects) (Beckmann et al., 2003; Woolrich et al., 2004).

We assessed statistical significance of group results using PALM (permutation analysis of linear models), a nonparametric permutation-based cluster-extent thresholding method, with 5000 random permutations and with cluster-forming threshold set to $z>2.3$ (Eklund et al., 2016; Winkler et al., 2014). We specifically used PALM because it allowed us to control for family structure, account for the effects of heritability that would make data points from related individuals non-independent, and restricts the number of time data can be permuted.

\section{Network Identification and Extraction}

To obtain an independent estimate of RSN connectivity during reward processing, we used the RSN maps from Smith et al. (2009) and ran spatial regression to extract whole-brain time courses associated with each RSN. Spatial regression was performed as part of dual-regression analysis (e.g. (Filippini et al., 2009; Gordon et al., 2012; Smith et al., 2015; Smith et al., 2014). The first step of the dual-regression analysis regresses the group spatial maps (RSNs in our case) on the functional data of each participant, and produces a time point by network matrix, which describes temporal behavior of each subject and network. The RSNs time course output from spatial regression was used to set up the PPI models (Figure 1). 


\section{Dual Regression: Network PPI Analysis}
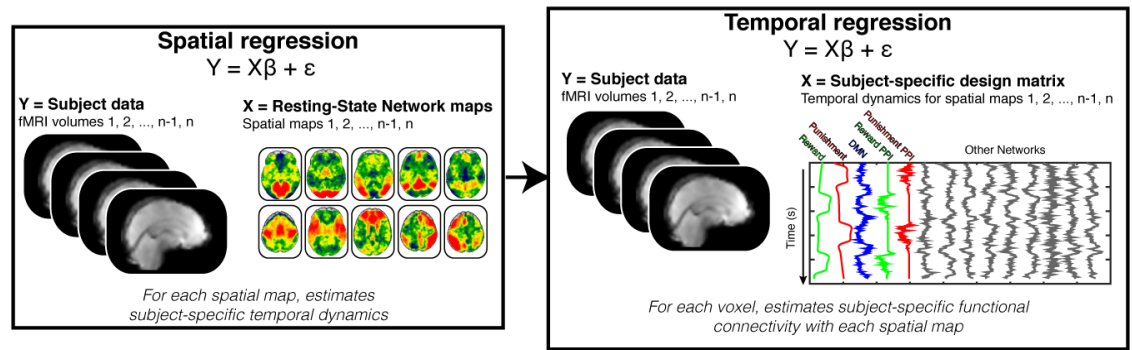

Figure 1: Analysis Schematic. Our network psychophysiological interaction analysis (nPPI) approach builds off of the dual-regression approach in that in consists of two key stages. In the first stage, the functional data from each participant is regressed onto a set of spatial maps reflecting common networks found in both resting-state and task-based data. This spatial regression results in a set of subject-specific temporal dynamics for each network. In the second stage, the temporal dynamics for each network are included in standard general linear model along with the task regressors (temporal regression). Regressors corresponding to psychophysiological interaction terms are created by multiplying each task regressor by the network of interest (i.e., default mode network).

\section{Behavioral data}

HCP provides rich behavioral data related to personality factors and cognition. However, we decided to focus on data related to impulsivity and personality as these constructs have been previously shown to be associated with PFC areas belonging to RSNs and striatal functioning (e.g. (Kunisato et al., 2011; Mitchell, 2011)) as well as with RSNs. To examine whether RSN connectivity during reward processing would show an association with cognitive and personality constructs we performed multiple regression analysis between behavioral measures obtained outside of the scanner and connectivity results. Specifically, we examined impulsivity as assessed by delayed discounting task and personality as assessed by Neuroticism/Extroversion/Openness Five Factor Inventory (NEO-FFI). We used area-under-thecurve discounting measure from the delayed discounting task as it provides a summary measure about the degree one discounts delayed rewards. From the NEO-FFI, a validated 
personality inventory (Costa \& McCrae, 2008), we used subscales that that assesses individual's degree of agreeableness, openness, conscientiousness, neuroticism, and extraversion. The regression included mean relative motion, NEO-FFI (agreeableness, openness, conscientiousness, neuroticism, extraversion), and discounting measures.

\section{Results}

\section{Reward-Related activation}

As a manipulation check, we examined whether there is differential activation in the striatum during reward processing. Consistent with prior studies using the card task (e.g., (Delgado et al., 2000; Tricomi et al., 2006), we observed that the receipt of reward in comparison to punishment, evoked greater activation in the striatum and other regions ( $p>0.001, z>3.1$, corrected for multiple comparisons; Table 1). This finding also furthers previously reported results from the HCP data (Barch et al., 2013). No regions survived the threshold during the punishment vs. reward comparison.

Table 1. Brain regions showing greater activation to reward vs. punishment.

Number of

\begin{tabular}{|c|c|c|c|c|c|c|}
\hline Region & Voxels & Hemisphere & Peak Z & Peak X & Peak Y & Peak Z \\
\hline Occipital Pole & 23088 & $\mathrm{R}$ & 16.1 & 14 & -96 & 18 \\
\hline Ventral Striatum & 4560 & $\mathrm{R}$ & 9.58 & 6 & 14 & -2 \\
\hline \multicolumn{7}{|c|}{ Dorsomedial Prefrontal } \\
\hline Cortex & 2266 & $\mathrm{~L}$ & 7.35 & -44 & 8 & 32 \\
\hline Supramarginal gyrus & 157 & $\mathrm{~L}$ & 4.66 & -50 & -48 & 16 \\
\hline \multicolumn{7}{|l|}{ Amygdala/parahippoca } \\
\hline mpal gyrus & 114 & $\mathrm{~L}$ & 4.75 & -30 & -10 & -14 \\
\hline \multicolumn{7}{|l|}{ Supplementary Motor } \\
\hline Area & 107 & $\mathrm{~L}$ & 5.1 & -6 & 6 & 56 \\
\hline
\end{tabular}




\section{Posterior Lobe,}

$\begin{array}{lllllll}\text { Cerebellum } & 83 & \mathrm{R} & 6.14 & 20 & -42 & -46 \\ \text { Paracingulate gyrus } & 65 & \mathrm{~L} & 4.34 & -8 & 24 & 44 \\ \text { Putamen } & 48 & \mathrm{R} & 4.96 & 28 & -12 & 10 \\ \text { Parahippocampal Gyrus } & 48 & \mathrm{~L} & 5.19 & -16 & -30 & -6 \\ \text { Parahippocampal Gyrus } & 39 & \mathrm{R} & 4.94 & 8 & -32 & -4 \\ \text { Middle Temporal Gyrus } & 39 & \mathrm{R} & 4.39 & 48 & -10 & -14 \\ \text { Caudate Tail } & 33 & \mathrm{R} & 4.49 & 22 & -18 & 24 \\ \text { Putamen } & 32 & \mathrm{R} & 4.77 & 32 & -10 & -10\end{array}$

We also examined how reward and punishment modulated responses of the DMN. To do this, we extracted the network responses using the spatial regression component of dual regression analysis (Fareri et al., 2020; Nickerson et al., 2017; Smith et al., 2015). These network responses were then regressed onto a model containing the predicted hemodynamic responses for reward and punishment blocks. Consistent with prior work, we found that the DMN was strongly deactivated during the task (Figure 2; $\mathrm{t}(453)=-75.31, \mathrm{p}<0.0001$ ), but the magnitude of deactivation did not significantly differ between reward and punishment blocks $(\mathrm{t}(453)=$ 1.35, $p=0.1792$ ). For completeness, we also assessed task-related responses in other networks that have been theorized to play and antagonist role with the DMN (Fox et al., 2005; Uddin et al., 2009). These networks included the executive control network (ECN) and the left and right frontoparietal networks (IFPN and rFPN). Each of these networks exhibited a positive response to the task $(E C N: \mathrm{t}(453)=2.37, \mathrm{p}=0.0184$; IFPN: $\mathrm{t}(453)=24.11, \mathrm{p}<0.0001 ;$ rFPN: $\mathrm{t}(453)=$ $37.57, p<0.0001$ ). Although the response to reward relative to punishment was larger in the $\operatorname{ECN}(t(453)=3.52, p=4.7838 \mathrm{e}-04)$ and the IFPN $(t(453)=4.71, p=3.3716 e-06)$, we found that the rFPN exhibited a larger response to punishment compared to reward $(t(453)=-2.55, p=$ 0.0110). While some reward-related processes have been shown to be lateralized in some contexts (Palminteri et al., 2009), such differences are beyond the scope of our paper, as they could be driven by the single-handed button-press response procedure in the HCP. 


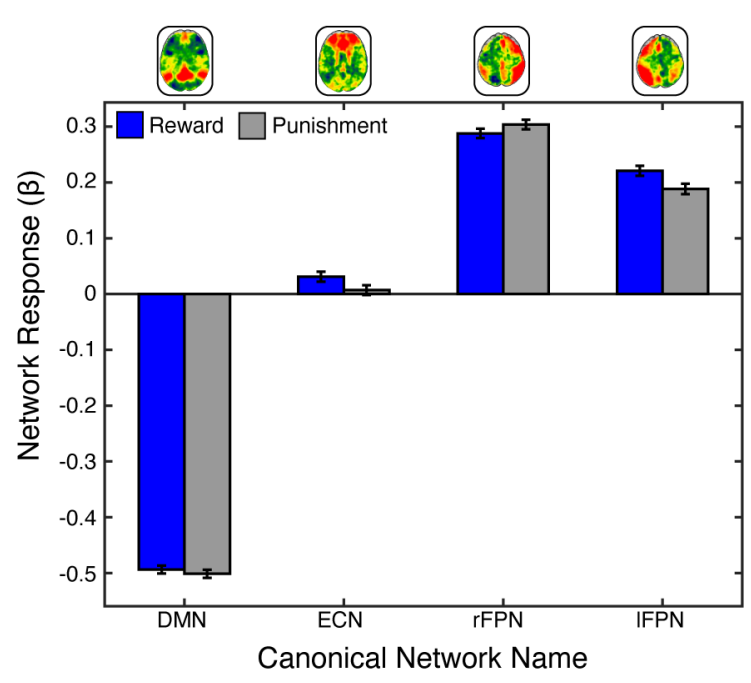

Figure 2. Network Responses to Reward and Punishment. We examined task-related responses for each network by regressing its activity onto a general linear model containing regressors for reward and punishment. We found that networks exhibited positive response to the task. As expected, we also found that the default mode network (DMN) was strongly deactivated during both reward and punishment. Abbreviations: $\mathrm{DMN}=$ default mode network; $\mathrm{ECN}=$ executive control network; rFPN = right fronto-parietal network; IFPN = left fronto-parietal network.

\section{Reward Enhances Coupling Between the DMN and Ventral Striatum}

Using the nPPI analysis, we examined reward-dependent connectivity of the DMN and the DMN showed increased connectivity with the left ventral striatum (VS) during the receipt of reward (relative to punishment) (Figure 3). Additionally, the DMN showed significant connectivity with the occipital pole and occipital fusiform gyrus. This result might suggest that the DMN is not simply a task-negative network but also has functional significance beyond selfreferential processing and interacts with other brain regions engaged in task performance (the striatum during reward processing in the current analysis). 


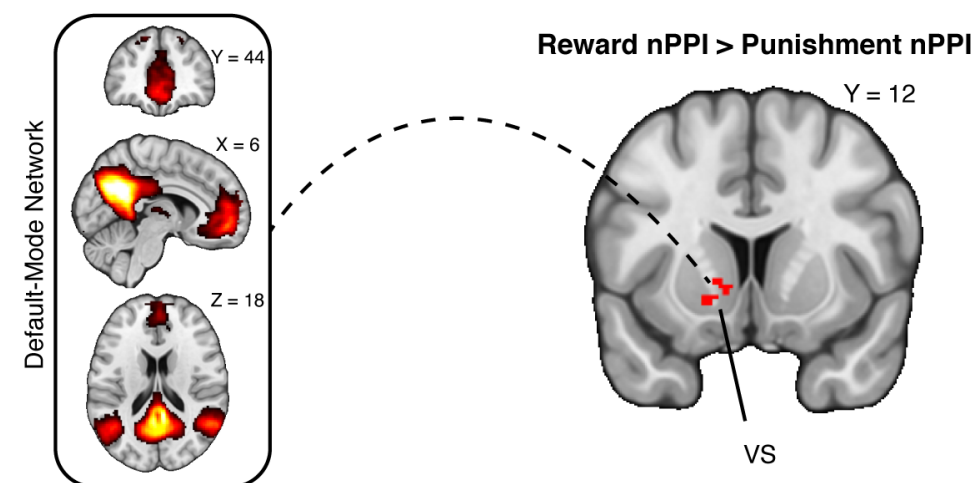

Figure 3: Enhanced Coupling between DMN and Ventral Striatum During Reward. We examined task-dependent changes with the DMN using a variant of psychophysiological interaction analyses. We found that an area of ventral striatum that exhibited increased connectivity with the DMN during reward relative to punishment.

Since the DMN has been shown to contribute to a spectrum of behaviors and psychological processes, we attempted to evaluate functional significance of DMN-VS connectivity by assessing whether discounting and personality measures are significant predictors of DMN-VS connectivity (Table 2). Results of the linear regression indicated that delayed discounting was not a significant predictor of DMN-VS connectivity. However, the constructs of openness $(\beta=.138, p=.004)$ and agreeableness $(\beta=-.124, p=.02)$ from NEO-FFI were significant predictors of DMN-VS connectivity. The NEO-FFI factor of openness has been shown to be related to sensation seeking and to reflect openness to experience, with low and high openness being characteristic of psychiatric disorders (Piedmont et al., 2009; Whiteside \& Lynam, 2001). While there has been substantial research into the relationship of impulsivity (as measured by delayed discounting) with brain activity and connectivity (Chen et al., 2017) (Hobkirk et al., 2019; Li et al., 2013), investigations of the relationship between personality characteristics and brain activity/connectivity are in its early stages.

Table 2. Multiple regression analysis results

\begin{tabular}{llll} 
Predictors & Beta & $t$ & $p$ \\
\hline NEO-FFI Agreeableness & -0.124 & -2.404 & 0.017 \\
NEO-FFI Openness to Experience & 0.138 & 2.881 & 0.004
\end{tabular}




\begin{tabular}{lrrr} 
NEO-FFI Conscientiousness & 0.043 & 0.803 & 0.422 \\
NEO-FFI Neuroticism & -0.045 & -0.841 & 0.401 \\
NEO-FFI Extraversion & 0.036 & 0.676 & 0.499 \\
Delay Discounting Area Under the Curve for \$200 & 0.031 & 0.48 & 0.631 \\
Delay Discounting Area Under the Curve for \$40,000 & -0.035 & -0.548 & 0.584 \\
Pos > Neg activation & 0.022 & 0.468 & 0.64 \\
Mean Relative Motion & -0.03 & -0.626 & 0.532 \\
\hline
\end{tabular}

We conducted additional analyses to evaluating the specificity of DMN findings by examining the connectivity between the VS other networks. Repeated measures ANOVA on connectivity parameters from each network showed the DMN to have the strongest connectivity with the VS $(F(453,1)=17,122, p<0.0001)$. Post-hoc paired t-tests revealed that the DMN-VS connectivity was significantly greater than ECN-VS connectivity $(t=3.3, p<.005), r F P N-$ VS connectivity $(t=5.8, p<.0001)$, and IFPN-VS connectivity $(t=5.8, p<.0001)$, pointing to the specificity of DMN-VS coupling during reward processing in comparison to other RSNs.

\section{Discussion}

With decision making and processing of action-outcome associations being vital in everyday life, it is imperative to understand the involvement of RSNs in these core processes, since they are purported to play an integral part in neural organization. Here, we examined the functional relationship between the DMN and processing of rewarding outcomes in a large sample of healthy individuals. Our findings demonstrate that the receipt of reward enhances connectivity between the DMN and the VS. This is consistent with a recent coordinate-based meta-analyses that documents an overlap between DMN and subjective value network regions that includes the VS (Acikalin et al., 2017). Further, increased DMN-VS connectivity is consistent with the nature of the card-guessing task. This task is not cognitively demanding, compared to a task that requires learning from rewards. Indeed, DMN has been shown to be engaged more during 
effortless/automatic decision-making (Vatansever et al., 2017). We might see greater connectivity between the ECN and the VS during a more effortful and cognitively demanding task, such as a learning task, where rewards reflect performance feedback (Denkova et al., 2019). The goal of the card-guessing task is to win monetary rewards for oneself. Thus, our results can be interpreted in light of the DMN being shown to be engaged in self-referential processing (Acikalin et al., 2017; Gusnard et al., 2001). Reward processing during the cardguessing task can fall under the umbrella of self-referential processing as subjects are winning monetary rewards for themselves. Thus, our findings point to a novel role for the DMN in reward processing and communication with the striatum, extending previous research about DMN connectivity and its role in behavior.

Our analysis also revealed that the strength to the DMN-VS connectivity during reward processing is positively associated with the personality factor of openness and agreeableness. While we did not have any a priori hypothesis about the directionality of the association between connectivity and personality, we speculate that this result contributes to the growing body of literature that investigates brain correlates of various personality types in both healthy (Beaty et al., 2018; Sampaio et al., 2014; Wei et al., 2014) and clinical populations (Takahashi et al., 2013). Further, recently openness has been shown to be associated with increased functional connectivity and dopamine release from the substantia nigra (Passamonti et al., 2014; Suridjan et al., 2012), a subcortical brain area that is heavily involved in reward processing and projects to the striatum (Haber \& Knutson, 2010).

Nevertheless, our results are also accompanied by limitations that merit further discussion. First, although our results may reflect a context-specific (reward receipt) modulation of effective connectivity from the DMN to the VS, such findings could also be explained by a modulation of stimulus-specific responses in the VS (Friston et al., 1997). Specifically, responses in the DMN may enhance the striatal response to reward without a change in connectivity. Future studies may be able to disambiguate these alternative explanations using dynamic causal modeling (Friston et al., 2003) or noninvasive brain stimulation (Polania et al., 2018). Second, it is not clear how our reported links between DMN-VS connectivity and openness would generalize to more diversified samples. Indeed, the HCP consists of healthy young adults, 
which may contribute to the limited range in openness scores and relatively small effect size in the brain-behavior correlations (Yarkoni, 2009). Third, our results are limited to the narrow construct of reward consumption. Examining other domains of reward processing-including anticipation (Knutson et al., 2000), learning (Daw et al., 2011), and valuation (Arulpragasam et al., 2018) - may reveal other patterns of DMN-VS connectivity or connectivity with other networks (Hallquist et al., 2018). In a similar vein, it will be important for future work to examine how DMN-VS connectivity during reward processing is modulated by contextual factors, such as perceived control (Wang \& Delgado, 2019), effort (Dobryakova et al., 2017), and social context (Fareri \& Delgado, 2014).

Despite these caveats, our results support the conclusion that DMN plays an active role in shaping responses in the VS during the execution of a reward processing task. These results broaden our conceptualization of DMN function by going beyond simplistic activation/deactivation schemes and show how responses in other brain regions are influenced by the engagement of DMN. More efforts to understand the dynamic interplay between the DMN and other brain regions could lead to a more integrative and parsimonious view of its function (Dohmatob et al., 2020). Such efforts could also help understand how the DMN contributes to clinical syndromes such as schizophrenia (Hu et al., 2017), depression (Posner et al., 2016), and substance abuse (Zhang \& Volkow, 2019), potentially providing new avenues for treatment and interventions. 


\section{Figure Captions}

\section{Figure 1: Analysis Schematic}

Our network psychophysiological interaction analysis (nPPI) approach builds off of the dualregression approach in that in consists of two key stages. In the first stage, the functional data from each participant is regressed onto a set of spatial maps reflecting common networks found in both resting-state and task-based data. This spatial regression results in a set of subject-specific temporal dynamics for each network. In the second stage, the temporal dynamics for each network are included in standard general linear model along with the task regressors (temporal regression). Regressors corresponding to psychophysiological interaction terms are created by multiplying each task regressor by the network of interest (i.e., default mode network).

\section{Figure 2: Network Responses to Reward and Punishment}

We examined task-related responses for each network by regressing its activity onto a general linear model containing regressors for reward and punishment. We found that networks exhibited positive response to the task. As expected, we also found that the default mode network (DMN) was strongly deactivated during both reward and punishment. Abbreviations: $\mathrm{DMN}$ = default mode network; $\mathrm{ECN}$ = executive control network; rFPN = right fronto-parietal network; IFPN = left fronto-parietal network.

\section{Figure 3: Enhanced Coupling between DMN and Ventral Striatum During Reward}

We examined task-dependent changes with the DMN using a variant of psychophysiological interaction analyses. We found that an area of ventral striatum that exhibited increased connectivity with the DMN during reward relative to punishment. 


\section{References}

Acikalin, M. Y., Gorgolewski, K. J., \& Poldrack, R. A. (2017). A Coordinate-Based Meta-Analysis of Overlaps in Regional Specialization and Functional Connectivity across Subjective Value and Default Mode Networks. Frontiers in Neuroscience, 11(1). doi:10.3389/fnins.2017.00001

Anticevic, A., Cole, M. W., Murray, J. D., Corlett, P. R., Wang, X.-J., \& Krystal, J. H. (2012). The role of default network deactivation in cognition and disease. Trends Cogn Sci, 16(12), 584-592. doi:https://doi.org/10.1016/j.tics.2012.10.008

Anticevic, A., Repovs, G., Shulman, G. L., \& Barch, D. M. (2010). When less is more: TPJ and default network deactivation during encoding predicts working memory performance. Neurolmage, 49(3), 2638-2648. doi:https://doi.org/10.1016/j.neuroimage.2009.11.008

Arulpragasam, A. R., Cooper, J. A., Nuutinen, M. R., \& Treadway, M. T. (2018). Corticoinsular circuits encode subjective value expectation and violation for effortful goal-directed behavior. Proceedings of the National Academy of Sciences, 115(22), E5233-E5242. doi:10.1073/pnas.1800444115

Barch, D. M., Burgess, G. C., Harms, M. P., Petersen, S. E., Schlaggar, B. L., Corbetta, M., . . Consortium, W. U.-M. H. (2013). Function in the human connectome: task-fMRI and individual differences in behavior. Neurolmage, 80, 169-189. doi:10.1016/j.neuroimage.2013.05.033

Beaty, R. E., Chen, Q., Christensen, A. P., Qiu, J., Silvia, P. J., \& Schacter, D. L. (2018). Brain networks of the imaginative mind: Dynamic functional connectivity of default and cognitive control networks relates to openness to experience. Hum Brain Mapp, 39(2), 811-821. doi:10.1002/hbm.23884

Beckmann, C. F., Jenkinson, M., \& Smith, S. M. (2003). General multilevel linear modeling for group analysis in FMRI. Neurolmage, 20(2), 1052-1063. doi:10.1016/S10538119(03)00435-X

Bluhm, R., Williamson, P., Lanius, R., Théberge, J., Densmore, M., Bartha, R., . . Osuch, E. (2009). Resting state default-mode network connectivity in early depression using a seed region-of-interest analysis: Decreased connectivity with caudate nucleus. 
Psychiatry and Clinical Neurosciences, 63(6), 754-761.

doi:https://doi.org/10.1111/j.1440-1819.2009.02030.x

Buckner, R. L., Andrews-Hanna, J. R., \& Schacter, D. L. (2008). The brain's default network: anatomy, function, and relevance to disease. Ann N Y Acad Sci, 1124, 1-38. doi:10.1196/annals.1440.011

Buckner, R. L., \& DiNicola, L. M. (2019). The brain's default network: updated anatomy, physiology and evolving insights. Nature Reviews Neuroscience, 20(10), 593-608.

Chen, Z., Guo, Y., \& Feng, T. (2017). Delay discounting is predicted by scale-free dynamics of default mode network and salience network. Neuroscience, 362, 219-227. doi:https://doi.org/10.1016/j.neuroscience.2017.08.028

Costa, P. T., \& McCrae, R. R. (2008). The revised NEO personality inventory (NEO-PI-R). The SAGE Handbook of Personality Theory and Assessment: Volume 2 - Personality Measurement and Testing, 179-198. doi:10.4135/9781849200479.N9

Dang, L. C., Donde, A., Madison, C., O'Neil, J. P., \& Jagust, W. J. (2012). Striatal Dopamine Influences the Default Mode Network to Affect Shifting between Object Features. J Cogn Neurosci, 24(9), 1960-1970. doi:10.1162/jocn_a_00252

Daw, N. D., Gershman, S. J., Seymour, B., Dayan, P., \& Dolan, R. J. (2011). Model-based influences on humans' choices and striatal prediction errors. Neuron, 69(6), 1204-1215.

Delgado, M. R., Nystrom, L. E., Fissell, C., Noll, D. C., \& Fiez, J. A. (2000). Tracking the hemodynamic responses to reward and punishment in the striatum. J Neurophysiol, 84(6), 3072-3077. Retrieved from http://www.ncbi.nlm.nih.gov/pubmed/11110834

Denkova, E., Nomi, J. S., Uddin, L. Q., \& Jha, A. P. (2019). Dynamic brain network configurations during rest and an attention task with frequent occurrence of mind wandering. Hum Brain Mapp, 40(15), 4564-4576. doi:10.1002/hbm.24721

Di, X., \& Biswal, B. B. (2014). Modulatory interactions between the default mode network and task positive networks in resting-state. PeerJ, 2, e367. doi:10.7717/peerj.367

Dobryakova, E., Jessup, R. K., \& Tricomi, E. (2017). Modulation of ventral striatal activity by cognitive effort. Neurolmage, 147(December 2016), 330-338.

doi:10.1016/j.neuroimage.2016.12.029 
Dobryakova, E., Rocca, M. A., \& Filippi, M. (2018). Cerebral Reorganization and Cognition in Multiple Sclerosis. In J. DeLuca \& B. M. Sandroff (Eds.), (pp. 368-368): APA AMERICAN PSYCHOLOGICAL ASSOCIATION.

Dohmatob, E., Dumas, G., \& Bzdok, D. (2020). Dark control: The default mode network as a reinforcement learning agent. Human Brain Mapping, 41(12), 3318-3341. doi:https://doi.org/10.1002/hbm.25019

Eklund, A., Nichols, T. E., \& Knutsson, H. (2016). Cluster failure: Why fMRI inferences for spatial extent have inflated false-positive rates. Proc Natl Acad Sci U S A, 113(28), 7900-7905. doi:10.1073/pnas.1602413113

Fareri, D. S., \& Delgado, M. R. (2014). Social Rewards and Social Networks in the Human Brain. Neuroscientist, 20(4), 387-402. doi:10.1177/1073858414521869

Fareri, D. S., Smith, D. V., \& Delgado, M. R. (2020). The influence of relationship closeness on default-mode network connectivity during social interactions. Soc Cogn Affect Neurosci, 15(3), 261-271.

Filippini, N., Maclntosh, B. J., Hough, M. G., Goodwin, G. M., Frisoni, G. B., Smith, S. M., . . Mackay, C. E. (2009). Distinct patterns of brain activity in young carriers of the APOEepsilon4 allele. Proc Natl Acad Sci U S A, 106(17), 7209-7214. doi:10.1073/pnas.0811879106

Fox, M. D., Snyder, A. Z., Vincent, J. L., Corbetta, M., Van Essen, D. C., \& Raichle, M. E. (2005). The human brain is intrinsically organized into dynamic, anticorrelated functional networks. Proc Natl Acad Sci U S A, 102(27), 9673-9678. doi:10.1073/pnas.0504136102

Friston, K. J., Buechel, C., Fink, G. R., Morris, J., Rolls, E., \& Dolan, R. J. (1997).

Psychophysiological and modulatory interactions in neuroimaging. Neurolmage, 6(3), 218-229. doi:10.1006/nimg.1997.0291

Friston, K. J., Harrison, L., \& Penny, W. (2003). Dynamic causal modelling. Neurolmage, 19(4), 1273-1302. doi:10.1016/s1053-8119(03)00202-7

Gignac, G. E., \& Szodorai, E. T. (2016). Effect size guidelines for individual differences researchers. Personality and Individual Differences, 102, 74-78. 
Glasser, M. F., Sotiropoulos, S. N., Wilson, J. A., Coalson, T. S., Fischl, B., Andersson, J. L., . . . Consortium, W. U.-M. H. (2013). The minimal preprocessing pipelines for the Human Connectome Project. Neurolmage, 80, 105-124. doi:10.1016/j.neuroimage.2013.04.127

Gordon, E. M., Stollstorff, M., \& Vaidya, C. J. (2012). Using spatial multiple regression to identify intrinsic connectivity networks involved in working memory performance. Hum Brain Mapp, 33(7), 1536-1552. doi:10.1002/hbm.21306

Gusnard, D. A., Akbudak, E., Shulman, G. L., \& Raichle, M. E. (2001). Medial prefrontal cortex and self-referential mental activity: relation to a default mode of brain function. Proc Natl Acad Sci U S A, 98(7), 4259-4264. doi:10.1073/pnas.071043098

Haber, S. N., \& Knutson, B. (2010). The reward circuit: linking primate anatomy and human imaging. Neuropsychopharmacology, 35(1), 4-26. doi:10.1038/npp.2009.129

Hallquist, M. N., Geier, C. F., \& Luna, B. (2018). Incentives facilitate developmental improvement in inhibitory control by modulating control-related networks. Neurolmage, 172, 369-380. doi:https://doi.org/10.1016/j.neuroimage.2018.01.045

Hasenkamp, W., Wilson-Mendenhall, C. D., Duncan, E., \& Barsalou, L. W. (2012). Mind wandering and attention during focused meditation: a fine-grained temporal analysis of fluctuating cognitive states. Neurolmage, 59(1), 750-760.

Hayden, B. Y., Smith, D. V., \& Platt, M. L. (2009). Electrophysiological correlates of default-mode processing in macaque posterior cingulate cortex. Proceedings of the National Academy of Sciences, 106(14), 5948-5953. doi:10.1073/pnas.0812035106

Hobkirk, A. L., Bell, R. P., Utevsky, A. V., Huettel, S., \& Meade, C. S. (2019). Reward and executive control network resting-state functional connectivity is associated with impulsivity during reward-based decision making for cocaine users. Drug and Alcohol Dependence, 194, 32-39. doi:https://doi.org/10.1016/j.drugalcdep.2018.09.013

Hu, M.-L., Zong, X.-F., Mann, J. J., Zheng, J.-J., Liao, Y.-H., Li, Z.-C., . . Tang, J.-S. (2017). A review of the functional and anatomical default mode network in schizophrenia. Neuroscience bulletin, 33(1), 73-84.

Hua, J. P. Y., Karcher, N. R., Merrill, A. M., O’Brien, K. J., Straub, K. T., Trull, T. J., \& Kerns, J. G. (2019). Psychosis risk is associated with decreased resting-state functional connectivity 
between the striatum and the default mode network. Cognitive, Affective, \& Behavioral Neuroscience, 19(4), 998-1011. doi:10.3758/s13415-019-00698-z

Hwang, J. W., Xin, S. C., Ou, Y. M., Zhang, W. Y., Liang, Y. L., Chen, J., . . Kong, J. (2016).

Enhanced default mode network connectivity with ventral striatum in subthreshold depression individuals. Journal of Psychiatric Research, 76, 111-120. doi:https://doi.org/10.1016/j.jpsychires.2016.02.005

Knutson, B., Westdorp, A., Kaiser, E., \& Hommer, D. (2000). FMRI visualization of brain activity during a monetary incentive delay task. Neurolmage, 12(1), 20-27. Retrieved from <Go to $|S|>: / / 000088317700003$

Kunisato, Y., Okamoto, Y., Okada, G., Aoyama, S., Nishiyama, Y., Onoda, K., \& Yamawaki, S. (2011). Personality traits and the amplitude of spontaneous low-frequency oscillations during resting state. Neuroscience Letters, 492(2), 109-113. doi:10.1016/j.neulet.2011.01.067

Li, N., Ma, N., Liu, Y., He, X.-S., Sun, D.-L., Fu, X.-M., . . Zhang, D.-R. (2013). Resting-State Functional Connectivity Predicts Impulsivity in Economic Decision-Making. The Journal of Neuroscience, 33(11), 4886-4895. doi:10.1523/jneurosci.1342-12.2013

McLaren, D. G., Ries, M. L., Xu, G., \& Johnson, S. C. (2012). A generalized form of contextdependent psychophysiological interactions (gPPI): A comparison to standard approaches. Neurolmage, 61(4), 1277-1286.

\section{doi:https://doi.org/10.1016/j.neuroimage.2012.03.068}

Mitchell, D. G. (2011). The nexus between decision making and emotion regulation: a review of convergent neurocognitive substrates. Behav Brain Res, 217(1), 215-231. doi:10.1016/j.bbr.2010.10.030

Nair, J., Klaassen, A.-L., Arato, J., Vyssotski, A. L., Harvey, M., \& Rainer, G. (2018). Basal forebrain contributes to default mode network regulation. Proceedings of the National Academy of Sciences, 115(6), 1352-1357. doi:10.1073/pnas.1712431115

Nickerson, L. D., Smith, S. M., Ongur, D., \& Beckmann, C. F. (2017). Using Dual Regression to Investigate Network Shape and Amplitude in Functional Connectivity Analyses. Front Neurosci, 11, 115. doi:10.3389/fnins.2017.00115 
Palminteri, S., Boraud, T., Lafargue, G., Dubois, B., \& Pessiglione, M. (2009). Brain Hemispheres Selectively Track the Expected Value of Contralateral Options. The Journal of Neuroscience, 29(43), 13465-13472. doi:10.1523/jneurosci.1500-09.2009

Park, H.-J., \& Friston, K. (2013). Structural and Functional Brain Networks: From Connections to Cognition. Science, 342(6158). doi:10.1126/science.1238411

Passamonti, L., Terracciano, A., Riccelli, R., Donzuso, G., Cerasa, A., Vaccaro, M. G., . . . Quattrone, A. (2014). Increased functional connectivity within mesocortical networks in open people. Neurolmage, 104, 301-309. doi:10.1016/j.neuroimage.2014.09.017

Piedmont, R. L., Sherman, M. F., Sherman, N. C., Dy-Liacco, G. S., \& Williams, J. E. G. (2009). Using the five-factor model to identify a new personality disorder domain: The case for experiential permeability. Journal of Personality and Social Psychology, 96(6), 12451258. doi:10.1037/a0015368

Polania, R., Nitsche, M. A., \& Ruff, C. C. (2018). Studying and modifying brain function with noninvasive brain stimulation. Nat Neurosci, 21(2), 174-187.

Poldrack, R. A., Baker, C. I., Durnez, J., Gorgolewski, K. J., Matthews, P. M., Munafo, M. R., . . . Yarkoni, T. (2017). Scanning the horizon: towards transparent and reproducible neuroimaging research. Nat Rev Neurosci, 18(2), 115-126. doi:10.1038/nrn.2016.167

Posner, J., Cha, J., Wang, Z., Talati, A., Warner, V., Gerber, A., .. . Weissman, M. (2016). Increased Default Mode Network Connectivity in Individuals at High Familial Risk for Depression. Neuropsychopharmacology, 41(7), 1759-1767. doi:10.1038/npp.2015.342

Pruim, R. H., Mennes, M., van Rooij, D., Llera, A., Buitelaar, J. K., \& Beckmann, C. F. (2015). ICAAROMA: A robust ICA-based strategy for removing motion artifacts from $\mathrm{fMRI}$ data. Neurolmage, 112, 267-277. doi:10.1016/j.neuroimage.2015.02.064

Raichle, M. E., MacLeod, A. M., Snyder, A. Z., Powers, W. J., Gusnard, D. A., \& Shulman, G. L. (2001). A default mode of brain function. Proceedings of the National Academy of Sciences, 98(2), 676-682.

Sampaio, A., Soares, J. M., Coutinho, J., Sousa, N., \& Gonçalves, Ó. F. (2014). The Big Five default brain: functional evidence. Brain structure \& function, 219(6), 1913-1922. doi:10.1007/s00429-013-0610-y 
Scheibner, H. J., Bogler, C., Gleich, T., Haynes, J.-D., \& Bermpohl, F. (2017). Internal and external attention and the default mode network. Neurolmage, 148, 381-389.

Smith, D. V., Gseir, M., Speer, M. E., \& Delgado, M. R. (2016). Toward a cumulative science of functional integration: A meta-analysis of psychophysiological interactions. Hum Brain Mapp, 37(8), 2904-2917. doi:10.1002/hbm.23216

Smith, D. V., Sip, K. E., \& Delgado, M. R. (2015). Functional connectivity with distinct neural networks tracks fluctuations in gain/loss framing susceptibility. Hum Brain Mapp, 36(7), 2743-2755. doi:10.1002/hbm.22804

Smith, D. V., Utevsky, A. V., Bland, A. R., Clement, N., Clithero, J. A., Harsch, A. E., ... Huettel, S. A. (2014). Characterizing individual differences in functional connectivity using dualregression and seed-based approaches. Neurolmage, 95, 1-12. doi:10.1016/j.neuroimage.2014.03.042

Smith, S. M., Fox, P. T., Miller, K. L., Glahn, D. C., Fox, P. M., Mackay, C. E., . . Beckmann, C. F. (2009). Correspondence of the brain's functional architecture during activation and rest. Proc Natl Acad Sci U S A, 106(31), 13040-13045. doi:10.1073/pnas.0905267106

Suridjan, I., Boileau, I., Bagby, M., Rusjan, P. M., Wilson, A. A., Houle, S., \& Mizrahi, R. (2012). Dopamine response to psychosocial stress in humans and its relationship to individual differences in personality traits. Journal of Psychiatric Research, 46(7), 890-897. doi:10.1016/j.jpsychires.2012.03.009

Takahashi, M., Shirayama, Y., Muneoka, K., Suzuki, M., Sato, K., \& Hashimoto, K. (2013). Low Openness on the Revised NEO Personality Inventory as a Risk Factor for TreatmentResistant Depression. PLoS One, 8(9), e71964-e71964. doi:10.1371/journal.pone.0071964

Tomasi, D., Volkow, N. D., Wang, R., Telang, F., Wang, G.-J., Chang, L., . . Fowler, J. S. (2009). Dopamine Transporters in Striatum Correlate with Deactivation in the Default Mode Network during Visuospatial Attention. PLoS One, 4(6), e6102. doi:10.1371/journal.pone.0006102 
Tricomi, E., Delgado, M. R., McCandliss, B. D., McClelland, J. L., \& Fiez, J. A. (2006). Performance feedback drives caudate activation in a phonological learning task. J Cogn Neurosci, 18(6), 1029-1043. doi:10.1162/jocn.2006.18.6.1029

Uddin, L. Q., Clare Kelly, A. M., Biswal, B. B., Xavier Castellanos, F., \& Milham, M. P. (2009). Functional connectivity of default mode network components: Correlation, anticorrelation, and causality. Human Brain Mapping, 30(2), 625-637. doi:https://doi.org/10.1002/hbm.20531

Utevsky, A. V., Smith, D. V., Young, J. S., \& Huettel, S. A. (2017). Large-Scale Network Coupling with the Fusiform Cortex Facilitates Future Social Motivation. eneuro, 4(5), ENEURO.0084-0017.2017. doi:10.1523/eneuro.0084-17.2017

Vatansever, D., Menon, D. K., Manktelow, A. E., Sahakian, B. J., \& Stamatakis, E. A. (2015). Default mode network connectivity during task execution. Neurolmage, 122, 96-104. doi:https://doi.org/10.1016/j.neuroimage.2015.07.053

Vatansever, D., Menon, D. K., \& Stamatakis, E. A. (2017). Default mode contributions to automated information processing. Proceedings of the National Academy of Sciences, 114(48), 12821-12826.

Wang, K. S., \& Delgado, M. R. (2019). Corticostriatal Circuits Encode the Subjective Value of Perceived Control. Cerebral Cortex, 29(12), 5049-5060. doi:10.1093/cercor/bhz045

Wang, X., Li, F., Zheng, H., Wang, W., Zhang, W., Liu, Z., . . Chen, A. (2015). Breakdown of the striatal-default mode network loop in schizophrenia. Schizophr Res, 168(1), 366-372. doi:https://doi.org/10.1016/j.schres.2015.07.027

Wei, L., Duan, X., Zheng, C., Wang, S., Gao, Q., Zhang, Z., . . Chen, H. (2014). Specific frequency bands of amplitude low-frequency oscillation encodes personality. Human Brain Mapping, 35(1), 331-339. doi:10.1002/hbm.22176

Whiteside, S. P., \& Lynam, D. R. (2001). The Five-Factor Model and Impulsivity : Using a Structural Model of Personality to Understand Impulsivity The Five Factor Model and impulsivity : using a structural model of personality to understand impulsivity. Personality and Individual Differences, 30(June 2016), 669-689. doi:10.1016/S01918869(00)00064-7 
Whitfield-Gabrieli, S., \& Ford, J. M. (2012). Default mode network activity and connectivity in psychopathology. Annu Rev Clin Psychol, 8, 49-76. doi:10.1146/annurev-clinpsy-032511143049

Winkler, A. M., Ridgway, G. R., Webster, M. A., Smith, S. M., \& Nichols, T. E. (2014).

Permutation inference for the general linear model. Neurolmage, 92, 381-397. doi:10.1016/j.neuroimage.2014.01.060

Woolrich, M. W., Behrens, T. E., Beckmann, C. F., Jenkinson, M., \& Smith, S. M. (2004). Multilevel linear modelling for FMRI group analysis using Bayesian inference. Neurolmage, 21(4), 1732-1747. doi:10.1016/j.neuroimage.2003.12.023

Woolrich, M. W., Ripley, B. D., Brady, M., \& Smith, S. M. (2001). Temporal autocorrelation in univariate linear modeling of FMRI data. Neurolmage, 14(6), 1370-1386. doi:10.1006/nimg.2001.0931

Yarkoni, T. (2009). Big correlations in little studies: Inflated fMRI correlations reflect low statistical power-Commentary on Vul et al.(2009). Perspectives on psychological science, 4(3), 294-298.

Zhang, R., \& Volkow, N. D. (2019). Brain default-mode network dysfunction in addiction. Neurolmage, 200, 313-331. doi:https://doi.org/10.1016/j.neuroimage.2019.06.036 